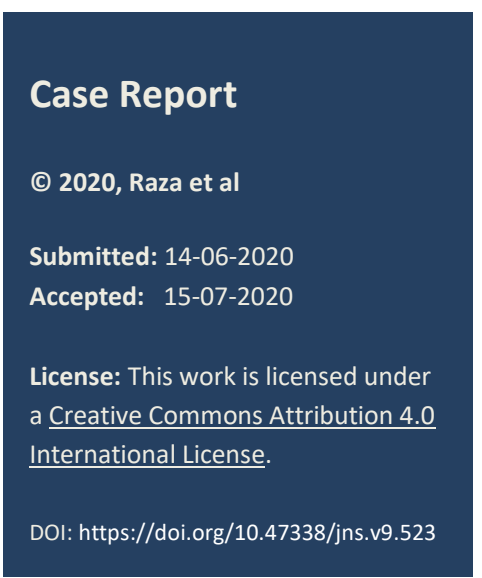

\title{
Congenital orbital teratoma: A case report
}

Ahmed Raza, ${ }^{1}$ Asma Mushtaq, ${ }^{1 *}$ Seema Qayyum, ${ }^{1}$ Fiza Azhar, ${ }^{1}$ Ahmad Imran, ${ }^{2}$ Ashvinah Qayyum, ${ }^{3}$

1 Department of Pediatric Ophthalmology, The Children's Hospital and The Institute of Child Health, Lahore

2 Department of Pediatric Radiology, The Children's Hospital and The Institute of Child Health, Lahore $3 \mathrm{CMH}$ Medical College, Lahore

Correspondence*: Dr. Asma Mushtaq, Department of Pediatric Ophthalmology, The Children's Hospital and the Institute of Child Health, Lahore. E-mail: drasmamushtaq18@gmail.com

\section{KEYWORDS}

Teratoma

Orbit

Proptosis

Neonate

\begin{abstract}
Background: Teratoma originates from all the three germinal layers and commonly found at sacrococcygeal region. The orbit is unusual site of occurrence, but they grow rapidly and cause massive proptosis.

Case Presentation: A 9-day-old female neonate presented with marked unilateral proptosis of the right eye. Imaging workup gave suspicion of a complex mass with internal hemorrhage or a teratoma. Modified exenteration was performed. Histopathological examination revealed mature cystic teratoma.
\end{abstract}

Conclusion: Orbital teratoma presents with marked disfiguring proptosis in neonatal life. Prompt complete surgical excision is curative in case of mature lesions.

\section{INTRODUCTION}

Teratomas are germ cell tumors containing derivatives of all germinal layers. The most common sites of occurrence are sacrococcygeal regions and gonads, with a female preponderance.[1] Teratoma occurring at the head and neck region are uncommon.[2]Usually the orbital teratoma are localized and benign; however, very few have malignant potential.[3-5]

The tumor grows rapidly, causing destructive proptosis and exposure keratopathy within days.[5] This leads to poor prognosis for vision or conservation of the globe in addition to considerable disfigurement. We report a case of mature orbital teratoma as it is exceptionally rare and challenging tumor.

\section{CASE REPORT}

A 9-day-old female neonate presented with massive proptosis of right eye since birth. She was first born child with unremarkable peri-natal and family history. On examination, right eye showed extensive proptosis. The eyeball was in the center and surrounded by the tumor (Fig.1A), making it impossible to distinguish the anatomical boundaries of the globe. There was severe conjunctival chemosis and corneal melting because of exposure keratopathy. Details of anterior and posterior segments were completely obscured. Left eye was un- remarkable. B-scan ultrasound of right eye showed calcifications in the vitreous. MRI showed a heterogonous density mass in right orbital cavity, with postcontrast enhancement displacing the globe inferiorly (Fig.2). Radiological diagnosis was a complex mass with internal hemorrhage or a teratoma.

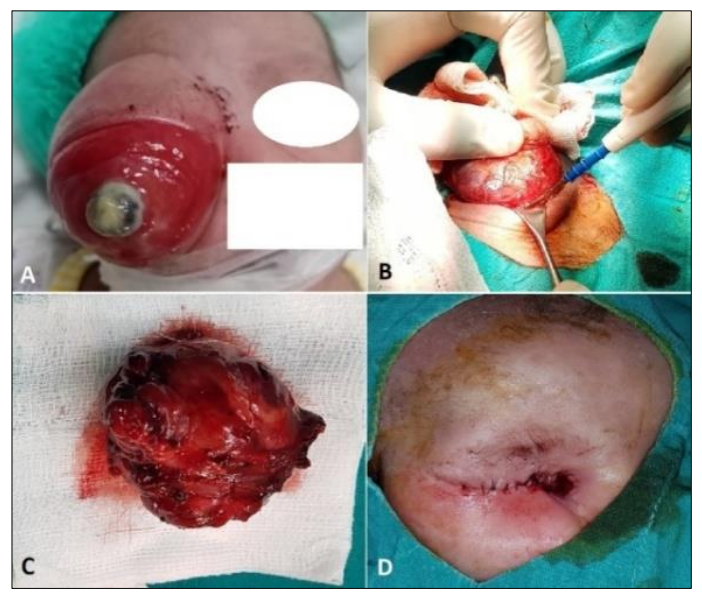

Figure 1: A) Proptosis with severe corneal melting. B) Lid sparing modified exenteration. C) Excised tumor. D) Partial lid closure done 6 weeks of first surgery.

Laboratory tests were within normal ranges. Lid sparing modified exenteration was performed (Fig. 1B, C) on 18 th day of life. An informed consent was obtained es- 
pecially mentioning postsurgical irreversible visual loss. Monopolar cautery was used to place a skin incision $2 \mathrm{~mm}$ above and below the upper and lower lash lines and extended just beyond both canthi.

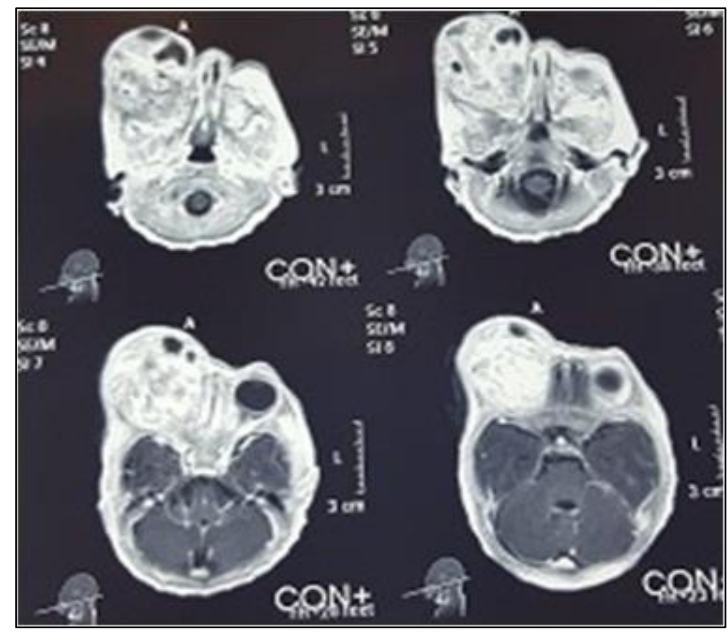

Figure 2: Axial T1 post contrast MRI: showing a heterogenous mass in right orbit with globe displacement.

The incision was passed through the skin and orbicularis oculi muscle, then undermining superiorly and inferiorly until the periosteum just outside the orbital rim was exposed for $360^{\circ}$.The periosteum was lifted with periosteal elevator for $360^{\circ}$ to expose the bony walls of orbital cavity. Homeostasis was achieved using cautery. The enucleation scissors were inserted between the periosteum and bone on the inferonasal side and slowly advanced to the orbital apex. The tissues were cut as close to the orbital apex as possible, and the orbital contents were removed by silk traction sutures in the eyelids. The residual adhesions were also excised.

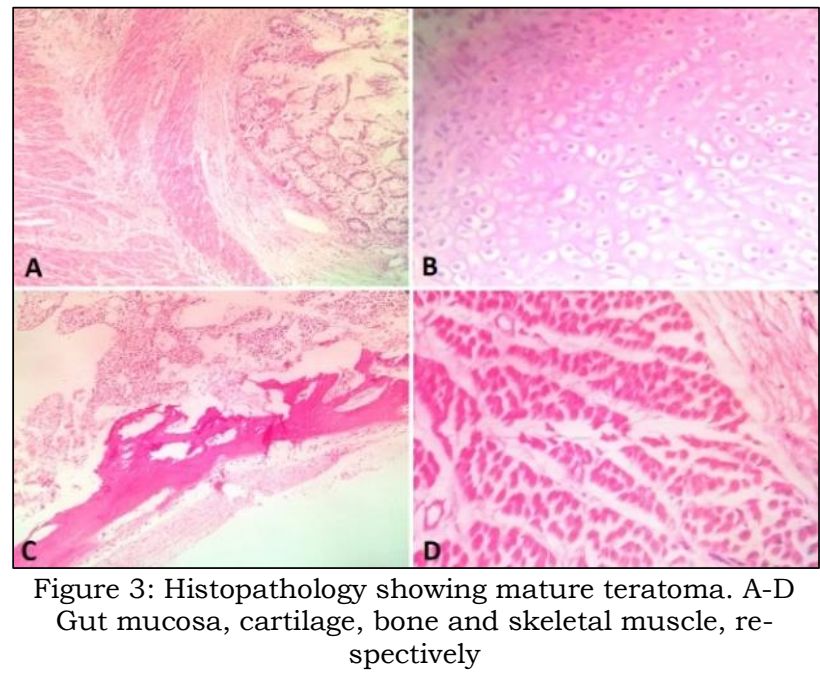

The empty socket was immediately packed with moist gauze for 10 minutes. Later, orbital apex was inspected for residual soft tissue that was removed piecemeal. The socket was packed with ointment-soaked gauze at the end. First dressing was changed after one week under general anesthesia. She was discharged with empty socket packed with dressing in good clinical condition on 10th postoperative day. She was kept on close follow-up. She had weekly dressing changed under general anesthesia (GA) in first month. Partial lid closure was done after 6 weeks of surgery to promote healing (Fig.1D). Later, the dressing was changed after every 2 weeks, without GA. Recovery was uneventful. Histological examination (Fig.3A-D) of the specimen showed mature teratoma (tumor composed of stratified squamous lining, skin adnexa, keratin, gut mucosa, muscle, cartilage, bone, adipose tissue, layers of retina, mesenchymal tissue, nerve, and ganglions). The patient was discussed in tumor board, but adjuvant chemotherapy was not suggested based on mature lesion. The patient is on our follow-up and we have planned for cosmetic rehabilitation once sufficient granulation tissue has healed the empty socket.

\section{DISCUSSION}

Congenital orbital teratoma is comprised of tissues from all three embryonic germ cell layers, and is categorized as mature or immature teratoma depending on the presence or absence of undifferentiated embryonic tissue, respectively.[6] Orbit is a rare (0.8\%) site of extra-gonadal teratoma with over 70 cases have been reported in literature.[7] Only one case of orbital teratoma has been reported from Pakistan in 2017.[8] The clinical suspicion in that case was retinoblastoma, although no radiological studies were performed. It was later confirmed as mature cystic teratoma on histopathology.

Kivelä and Tarkkanen classified orbital teratomas as: primary intraocular teratoma, primary orbital teratoma, combined orbital and extra orbital teratoma, and secondary orbital teratoma.[4]Our case was combined orbital and extra orbital teratoma. Clinically, orbital teratomas present in early days of life as proptosis. The tumor stretches recti muscles, retraction of palpebral aperture, leading to axial proptosis and exposure keratopathy as found in the index case. In some cases, eyeball may be normal and embedded deep inside the cystic mass. [4] However, the diagnosis may be delayed sometimes due to clinical features mimicking orbital cellulitis, causing significant visual morbidity.[2]

Radiological features of benign orbital teratomas include multi loculated cystic mass with focal calcifications and fat. Eyeball is compressed and optic nerve may be displaced. There is distension of orbital walls without their destruction. Ultrasound shows multiple areas of low and high reflectivity in addition to foci of calcification.[9] Retinoblastoma is an important cause intraocular calcification in this age group, so may be considered as a differential diagnosis.[10]

Management of congenital orbital teratoma is not straightforward. As tumor is rapidly progressive, salvaging vision is always challenging. Mee et al, were 
able to prevent useful vision after surgery in an 18month-old baby with inferior orbital teratoma, as eyeball was not destroyed by the tumor.[11] In our case, irreversible damage was caused by exposure keratopathy and corneal melting thus precluding vision salvage. Early surgical intervention was done. Although vision cannot be preserved in cases like the index case, but prompt surgery prevents further mutilating surgeries like radical exenteration.

Long term follow-up is required, initially to address the empty socket, and later for ocular cosmetic rehabilitation, and recurrence. In the index case, the patient was initially managed for empty socket with weekly dressings under GA and later partial lid closure was done to improve healing. Once sufficient granulation tissue fills the orbital cavity, orbital socket reconstruction will be done to fill the orbital volume using temporalis muscle rotational flap and skin graft, dermo-fat graft, and orbital implants. Light weight spectacle bound pros-

\section{REFERENCES}

1. Mann JR, Gray ES, Thornton C, Raafat F, Robinson K, Collins GS, et al. Mature and immature extracranial teratomas in children: The UK children's cancer study group experience. J Clin Oncol. 2008;26:3590-7.

2. Alkatan HM, Chaudhry I, Alayoubi A. Mature teratoma presenting as orbital cellulitis in a 5-month-old baby. Ann Saudi Med. 2013;33:623-6.

3. Alkatan HM, AlObaidanOS, KfouryH AlFakyYH.Orbital immature teratoma: A rare entity with diagnostic challenges. Saudi J Ophthalmol. 2018; 32:75-8.

4. Prakash MV, Indira R, Radhakrishnan M, Leela G. Malignant orbital teratoma in a neonate: A clinicopathological case report. J Postgrad Med. 2017;63:203-5

5. Ogun OA, Ogun GO, Brown BJ, Mosuro AL, Ashaye AO. Congenital orbital teratoma: a case report and challenges of its management in a resource limited setting. Pan Afr Med J. 2012;12:3. thesis will be given for a good cosmesis. Follow up with MRI scan and serum alpha fetoprotein levels (AFP) will be done twice a year to monitor for recurrence.

In conclusion, orbital teratoma is a rare site of occurrence of germ cell tumors. It must be considered in a newborn with rapid progressive proptosis. Early surgical intervention with eventual plan of ocular cosmetic rehabilitation is suggested for good outcome.

Acknowledgements: Nil

Conflict of Interest: None declared

Source of Support: Nil

Consent to Publication: Author(s) declared taking informed written consent for the publication of clinical photographs/material (if any used), from the legal guardian of the patient with an understanding that every effort will be made to conceal the identity of the patient, however it cannot be guaranteed.

Author Contributions: Author(s) declared to fulfil authorship criteria as devised by ICMJE and approved the final version.

6. Aiyub S, Chan WO, Szetu J, Sullivan LJ, Pater J, Cooper $\mathrm{P}$, et al. Congenital orbital teratoma. Indian $\mathrm{J}$ Ophthalmol. 2013;61:767-69.

7. Pellerano F, Guillermo E, Garrido G, Berges P. Congenital orbital teratoma. Ocul Oncol Pathol. 2017;3:11-6.

8. Gulzar R, Shahid R, Mirza T. Congenital orbital teratoma with unilateral proptosis. J Coll Phys Surg Pak. 2017; 27:61-2.

9. González C, Restrepo CA, Salazar GI, Monsalve P. Congenital orbital teratoma. Case report. Colomb Med. 2012;43:82-5.

10. Kachewar SG, Kulkarni DS.An Imaging review of intraocular calcifications. J Clin Diagn Res. 2014; 8:203-5.

11. Mee JJ, McKelvie PA, McNab AA. Orbital teratoma: late presentation with normal vision. Clin Experiment Ophthalmol. 2002; 30:41-3 